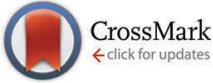

Cite this: Phys. Chem. Chem. Phys., 2015, 17, 19230

Received 17th April 2015, Accepted 17th June 2015

DOI: $10.1039 / \mathrm{c} 5 \mathrm{cp} 02248 \mathrm{~g}$

www.rsc.org/pccp

\title{
Distortions of ethyne when complexed with a cuprous or argentous halide: the rotational spectrum of $\mathrm{C}_{2} \mathrm{H}_{2} \ldots \mathrm{CuF} \dagger$
}

\author{
Daniel P. Zaleski, ${ }^{a}$ Susanna L. Stephens, ${ }^{a}$ David P. Tew, ${ }^{b}$ Dror M. Bittner, ${ }^{a}$ \\ Nicholas R. Walker*a and Anthony C. Legon*b
}

\begin{abstract}
A new molecule $\mathrm{C}_{2} \mathrm{H}_{2}$. . CuF has been synthesized in the gas phase by means of the reaction of laser-ablated metallic copper with a pulse of gas consisting of a dilute mixture of ethyne and sulfur hexafluoride in argon. The ground-state rotational spectrum was detected by two types of Fourier-transform microwave spectroscopy, namely that conducted in a microwave Fabry-Perot cavity and the chirped-pulse broadband technique. The spectroscopic constants of the six isotopologues ${ }^{12} \mathrm{C}_{2} \mathrm{H}_{2} \cdots{ }^{63} \mathrm{Cu}^{19} \mathrm{~F},{ }^{12} \mathrm{C}_{2} \mathrm{H}_{2} \cdots{ }^{65} \mathrm{Cu}^{19} \mathrm{~F}$, ${ }^{13} \mathrm{C}_{2} \mathrm{H}_{2} \cdot{ }^{63} \mathrm{Cu}^{19} \mathrm{~F},{ }^{13} \mathrm{C}_{2} \mathrm{H}_{2} \cdot{ }^{65} \mathrm{Cu}^{19} \mathrm{~F},{ }^{12} \mathrm{C}_{2} \mathrm{D}_{2} \cdot{ }^{63} \mathrm{Cu}^{19} \mathrm{~F}$ and ${ }^{12} \mathrm{C}_{2} \mathrm{D}_{2} \cdot{ }^{65} \mathrm{Cu}^{19} \mathrm{~F}$ were determined and interpreted to show that the molecule has a planar, T-shaped geometry belonging to the molecular point group $C_{2 v}$, with CuF forming the stem of the T. Quantitative interpretation reveals that the ethyne molecule is distorted when subsumed into the complex in such manner that the $\mathrm{C} \equiv \mathrm{C}$ bond lengthens (by $\delta r$ ) and the two $\mathrm{H}$ atoms cease to be collinear with the $\mathrm{C} \equiv \mathrm{C}$ internuclear line. The $\mathrm{H}$ atoms move symmetrically away from the approaching $\mathrm{Cu}$ atom of $\mathrm{CuF}$, to increase each $* \equiv \mathrm{C}-\mathrm{H}$ angle by $\delta A=14.65(2)^{\circ}$, from $180^{\circ}$ to $194.65(2)^{\circ}$. $A b$ initio calculations at the explicitly-correlated level of theory $\operatorname{CCSD}(\mathrm{T})\left(\mathrm{F} 12^{*}\right) /$ aug-cc-pVTZ lead to good agreement with the experimental geometry. It is shown that similar distortions $\delta r$ and $\delta A$, similarly determined, for four complexes $\mathrm{C}_{2} \mathrm{H}_{2} \ldots \mathrm{MX}(\mathrm{M}=\mathrm{Cu}$ or $\mathrm{Ag} ; \mathrm{X}=\mathrm{F}, \mathrm{Cl}$ or $\mathrm{CCH})$ are approximately linearly related to the energies $D_{e}$ for the dissociation process $\mathrm{C}_{2} \mathrm{H}_{2} \cdots M X=\mathrm{C}_{2} \mathrm{H}_{2}+\mathrm{MX}$.
\end{abstract}

\section{Introduction}

The coordination of transition metal atoms $\mathrm{M}$, cations $\mathrm{M}^{+}$or polar salts $\mathrm{M}^{+} \mathrm{X}^{-}$to alkynes and alkenes has been described in terms of the interaction of the $\pi$ electrons of the latter compounds with the $\pi^{*}$ orbitals of the metal atom or ion. ${ }^{1,2}$ Such interactions achieve importance by virtue of their presence in coordination compounds that feature in hydrogenation and polymerization reactions. ${ }^{3}$ For that reason, we have recently been investigating systematically a series of simple, gas-phase complexes of the type $\mathrm{C}_{2} \mathrm{H}_{2} \cdots \mathrm{MX}$ which contain such interactions, where $\mathrm{M}$ is a coinage metal atom and $\mathrm{X}$ is a halogen atom. ${ }^{4-6}$ The advantage of working in the gas phase is that distortions of the alkyne on complex formation are free from lattice or solvent effects and are therefore intrinsic to the interaction of interest. The technique employed in the synthesis of $\mathrm{C}_{2} \mathrm{H}_{2} \cdots \mathrm{MX}$ complexes is laser ablation of the metal $\mathrm{M}$ in the

\footnotetext{
${ }^{a}$ School of Chemistry, Newcastle University, Bedson Building, Newcastle-upon-Tyne, NE1 TRU, UK. E-mail: nick.walker@newcastle.ac.uk

${ }^{b}$ School of Chemistry, University of Bristol, Cantock's Close, Bristol, BS8 1TS, UK. E-mail: a.c.legon@bristol.ac.uk

$\dagger$ Electronic supplementary information (ESI) available. See DOI: 10.1039/ c5cp02248g
}

presence of both ethyne and a source of halogen atom $\mathrm{X}$ in a preponderating excess of argon gas. The subsequent, rapid supersonic expansion of the mixture into a vacuum cools and stabilizes the product molecules and allows their rotational spectra to be observed. By these methods it has been possible to determine, with all the precision associated with microwave spectroscopy, the geometries of several species ${ }^{4-6} \mathrm{C}_{2} \mathrm{H}_{2} \cdots \mathrm{MX}$, where $\mathrm{MX}$ is $\mathrm{AgCl}, \mathrm{AgCCH}$, or $\mathrm{CuCl}$.

In this article, we report the rotational spectra of six isotopologues of $\mathrm{C}_{2} \mathrm{H}_{2} \cdots \mathrm{Cu}-\mathrm{F}$. The complexes were produced by the laser ablation of metallic copper in the presence of a pulse of ethyne- $\mathrm{SF}_{6}-\mathrm{Ar}$ gas mixture and their spectra were detected by two versions of the Fourier-transform microwave technique. Interpretation of the spectroscopic constants thereby determined leads to a precise characterization of the distortion that ethyne undergoes when it is coordinated to CuF. The distortions will be compared with those predicted by an $a b$ initio calculation conducted at the $\operatorname{CCSD}(\mathrm{T})\left(\mathrm{F} 12^{*}\right) / \mathrm{AVTZ}$ level of theory Such distortions have now been established, both experimentally and $a b$ initio, as accompanying the attachment of $\mathrm{C}_{2} \mathrm{H}_{2}$ to $\mathrm{MX}=\mathrm{CuF}, \mathrm{CuCl}, \mathrm{AgCl}$ and $\mathrm{AgCCH}$ (where $\mathrm{M}$ is copper or silver, and $\mathrm{X}=\mathrm{F}, \mathrm{Cl}$ or $\mathrm{CCH}$ as appropriate). Variations in these distortions with (1) M and (2) X will be considered. 


\section{Experimental and theoretical methods}

Experimental data for $\mathrm{C}_{2} \mathrm{H}_{2} \cdots \mathrm{CuF}$ and $\mathrm{C}_{2} \mathrm{D}_{2} \cdots \mathrm{CuF}$ were recorded on a chirped-pulse Fourier transform microwave spectrometer ${ }^{7}$ operating between 7 and $18.5 \mathrm{GHz}$. The instrument has been previously described in detail. ${ }^{8,9} \mathrm{~A}$ gas sample was prepared containing approximately $1 \% \mathrm{C}_{2} \mathrm{H}_{2}$ (or $\mathrm{C}_{2} \mathrm{D}_{2}$ as appropriate) and $1 \%$ $\mathrm{SF}_{6}$ balanced in 6 bar argon. The gas mixture then pulsed over a copper target rod which was ablated with a Nd:YAG laser. The gas pulse was subsequently expanded into a vacuum chamber. For these experiments, the pulsed nozzle was placed perpendicular to the axis of microwave propagation. During each sample injection cycle, 8 free induction decays (FID's) were measured, each $20 \mu \mathrm{s}$ in duration. For $\mathrm{C}_{2} \mathrm{H}_{2} \cdots \mathrm{CuF}$ a total of $480 \mathrm{k}$ FID's were acquired $(16 \mathrm{~h})$, for $\mathrm{C}_{2} \mathrm{D}_{2} \cdots \mathrm{CuF}$ the total was $780 \mathrm{k}(26 \mathrm{~h})$, and $720 \mathrm{k}$ $(24 \mathrm{~h})$ for ${ }^{13} \mathrm{C}_{2} \mathrm{H}_{2}-\mathrm{CuF}$. In each case, Fourier transformation used a digital Kaiser-Bessel window function.

For ${ }^{12} \mathrm{C}_{2} \mathrm{H}_{2} \cdots \mathrm{CuF}$, data were also recorded on a Balle-Flygare cavity spectrometer, ${ }^{10,11}$ with an equivalent gas sample. These measurements were acquired with the pulsed nozzle placed coaxial with the direction of microwave propagation. Transitions measured in this arrangement appear as Doppler doublets, each normally with a full-width half maximum (FWHM) of $5 \mathrm{kHz}$ (but see Section 3.1); this is compared to a FWHM of approximately $150 \mathrm{kHz}$ for the perpendicular arrangement found in the broadband instrument. Only samples containing isotopes in natural abundance were employed when using this spectrometer.

Geometry optimizations were performed using $\operatorname{CCSD}(\mathrm{T})\left(\mathrm{F} 12^{*}\right),{ }^{12}$ a coupled-cluster method with single and double excitations, explicit correlation, ${ }^{13}$ and a perturbative treatment of triple excitations. ${ }^{14}$ An AVTZ basis set combination was used, by which we mean that the aug-cc-pVTZ basis sets ${ }^{15}$ were used for the $\mathrm{C}, \mathrm{F}$ and $\mathrm{H}$ atoms and the aug-cc-pVTZ-PP basis for $\mathrm{Cu}$, in combination with the ECP-10-MDF effective core potential on $\mathrm{Cu}$ to account for scalar relativistic effects. ${ }^{16,17}$ The frozen-core approximation was used throughout, and all calculations were performed using the MOLPRO package. ${ }^{18}$ The equilibrium dissociation energy $D_{\mathrm{e}}$ for the process $\mathrm{C}_{2} \mathrm{H}_{2} \cdots \mathrm{Cu}-\mathrm{F}=\mathrm{C}_{2} \mathrm{H}_{2}+\mathrm{Cu}-\mathrm{F}$ was also computed at the $\operatorname{CCSD}(\mathrm{T})-$ (F12*)/AVTZ level using the counterpoise correction method ${ }^{19}$ where, for numerical stability, the CABS singles correction was not included in the correction term. The result was $D_{\mathrm{e}}=170 \mathrm{~kJ} \mathrm{~mol}^{-1}$.

For the free $\mathrm{CuF}$, the bond length was optimised on a series of potential curves including successive corrections for core correlation, computed at the CCSD(T) level by using the cc-pwCVQZ basis, full triples at the frozen-core level with a cc-pVQZ basis and perturbative quadruples using the cc-pVTZ basis, following a similar procedure to that employed by Gauss et $a l^{20}$ Post $\operatorname{CCSD}(\mathrm{T})$ calculations were performed using the MRCC program ${ }^{21}$ and yielded the fully corrected bond length $r_{\mathrm{e}}(\mathrm{Cu}-\mathrm{F})=1.7430 \AA$, with a basis set uncertainty of $0.0006 \AA$. This result is in good agreement with the experimental value $1.74493 \AA$ calculated from equilibrium rotational constants ${ }^{22}$ of ${ }^{63} \mathrm{Cu}^{19} \mathrm{~F}$ and ${ }^{65} \mathrm{Cu}^{19} \mathrm{~F}$ by using the expression $r_{\mathrm{e}}=\left\{h / 8 \pi^{2} \mu B_{\mathrm{e}}\right\}^{\frac{1}{2}}$. At the $\operatorname{CCSD}(\mathrm{T})\left(\mathrm{F} 12^{*}\right) /$ aug-cc-pVTZ level of theory, as used for $\mathrm{C}_{2} \mathrm{H}_{2} \cdots \mathrm{Cu}-\mathrm{F}$, the equilibrium bond length for the free $\mathrm{CuF}$ molecule is predicted to be $1.7422(25) \AA$, where the error is that estimated (via gaussian error propagation) to arise from basis set incompleteness $(0.0007 \AA)$, core valence correlation error $(0.0021 \AA)$ and higher-order correlation $(0.0011 \AA)$.

\section{Results}

\subsection{Determination of spectroscopic constants}

For all six isotopologues investigated, only vibrational groundstate, a-type R-branch transitions $(J+1)_{K_{-1} K_{1}^{\prime}} \rightarrow J_{K_{-1} K_{1}^{\prime \prime}}$ with $K_{-1}=0$ or 1 of the asymmetric-rotor complex $\mathrm{C}_{2} \mathrm{H}_{2} \cdots \mathrm{Cu}-\mathrm{F}$ were observed under the experimental conditions described in Section 2. Each transition carried a resolvable nuclear quadrupole hyperfine structure resulting from a single quadrupolar nucleus ${ }^{63} \mathrm{Cu}$ or ${ }^{65} \mathrm{Cu}$ $(I=3 / 2)$. Transition frequencies of each isotopologue were fitted by means of the program PGOPHER ${ }^{23}$ with the following choice of Hamiltonian:

$$
H=H_{\mathrm{R}}-\frac{1}{6} \mathbf{Q}(\mathrm{Cu}): \nabla \mathbf{E}(\mathrm{Cu}),
$$

in which $\mathbf{Q}(\mathrm{Cu})$ and $\nabla \mathbf{E}(\mathrm{Cu})$ are the $\mathrm{Cu}$ nuclear electric quadrupole tensor and the electric field gradient tensor at the $\mathrm{Cu}$ nucleus, respectively. $H_{\mathrm{R}}$ is the familiar Hamiltonian for a semi-rigid, asymmetric-rotor molecule and contains both rotational constants and centrifugal distortion constants. The $H$ matrix was constructed in the coupled symmetric rotor basis, with the Watson A reduction ${ }^{24}$ chosen for $H_{\mathrm{R}}$, In addition to the rotational constants, $H_{\mathrm{R}}$ contains terms involving the five quartic centrifugal distortion constants $\Delta_{J}, \Delta_{J K}, \Delta_{K}, \delta_{J}$, and $\delta_{K}$.

The rotational constants of the most abundant isotopologue ${ }^{12} \mathrm{C}_{2} \mathrm{H}_{2} \cdots{ }^{63} \mathrm{Cu}^{19} \mathrm{~F}$ are large enough that only one group of $J+1 \rightarrow J$ transitions, namely that with $J=1$, could be measured in the frequency range available. A set of internally consistent arguments will be presented to show that the geometry of $\mathrm{C}_{2} \mathrm{H}_{2} \cdots \mathrm{Cu}-\mathrm{F}$ is of the planar, T-shaped type in which the non-covalent interaction of the two component molecules involves primarily the $\mathrm{Cu}$ atom of $\mathrm{CuF}$ and the $\pi$ bond of ethyne, as shown in Fig. 1. The experimental consequences of such a geometry are as follows:

(1) The molecule is a nearly prolate, planar asymmetric rotor of molecular point group $C_{2 \mathrm{v}}$, with the $a$ axis coincident with the $C_{2}$ axis. The rotational constant $A_{0}$ is close in magnitude to the rotational constant $B_{0}$ of free ethyne. Any difference of the two rotational constants will provide quantitative information about the extent of any geometrical distortion of the ethyne molecule when subsumed into $\mathrm{C}_{2} \mathrm{H}_{2} \cdots \mathrm{Cu}-\mathrm{F}$.

(2) The ground-state molecule will have a small positive inertia defect

$$
\Delta_{0}=I_{c}^{0}-I_{b}^{0}-I_{a}^{0},
$$

which is an important criterion of molecular planarity.

(3) A rotation $C_{2}^{a}$ exchanges a pair of equivalent protons $(I=1 / 2)$ and this endows $K_{-1}=1$ transitions with a nuclear spin statistical weight of 3 relative to that of 1 for $K_{-1}=0$ transitions. This effect is evident in the set of $2_{12} \rightarrow 1_{11}, 2_{02} \rightarrow 1_{01}$, $2_{11} \rightarrow 1_{10}$ transitions, as can be seen from the recording of these transitions shown in Fig. 2. Similar arguments applied to 


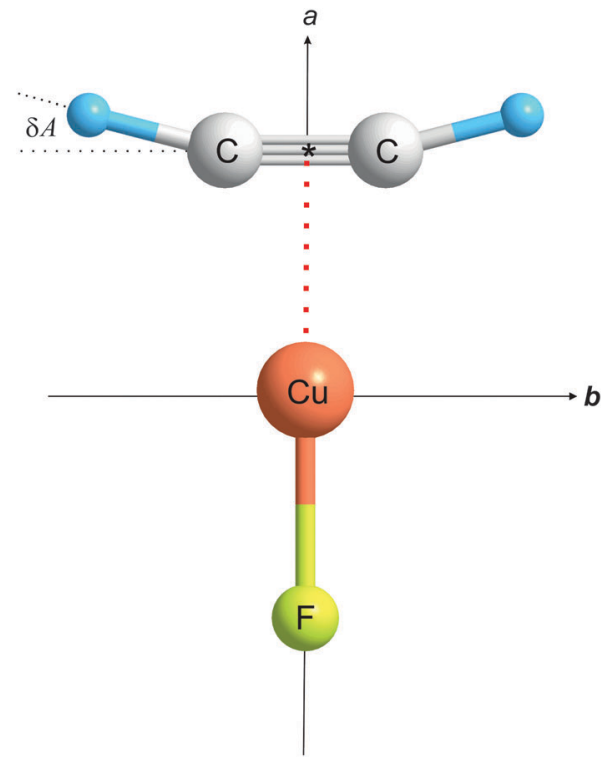

Fig. 1 The geometry (drawn to scale) of $\mathrm{C}_{2} \mathrm{H}_{2} \cdots \mathrm{Cu}-\mathrm{F}$ determined from analysis of the rotational spectra of six isotopologues. The atoms are coplanar and the principal inertia axis a coincides with the $C_{2}$ axis of symmetry. The ethyne subunit is significantly distorted relative to the free molecule, both in respect of the angle $A$ and of the distance $r(\mathrm{C} \equiv \mathrm{C})$. See text for discussion of the detailed geometry.

the ${ }^{12} \mathrm{C}_{2} \mathrm{D}_{2} \cdots \mathrm{Cu}^{19} \mathrm{~F}$ and ${ }^{13} \mathrm{C}_{2} \mathrm{H}_{2} \cdots \mathrm{Cu}^{19} \mathrm{~F}$ isotopologues require the corresponding ratios to be $1: 2$ and $6: 10$, respectively, which were indeed as observed.

(4) Isotopic substitution of ${ }^{63} \mathrm{Cu}$ by ${ }^{65} \mathrm{Cu}$ should leave $A_{0}$ unchanged.

It is evident that the rotational constant $A_{0}$ has a central role to play in drawing conclusions about the symmetry and geometry of $\mathrm{C}_{2} \mathrm{H}_{2} \cdots \mathrm{Cu}-\mathrm{F}$. Unfortunately, the $(J+1)_{K_{-1} K_{1}^{\prime}} \rightarrow J_{K_{-1} K_{1}^{\prime \prime}}$ $\left(K_{-1}=0\right.$ or 1$)$ transition frequencies have a very weak dependence on $A_{0}$ and it was not possible from the measured transition frequencies to determine $A_{0}$ independently of the two centrifugal distortion constants $\Delta_{J}$ and $\Delta_{J K}$. (There is insignificant dependence on the remaining centrifugal distortion constants.) This difficulty was circumvented by the following approach.

It has been shown elsewhere that centrifugal distortion constants of molecules similar to $\mathrm{C}_{2} \mathrm{H}_{2} \cdots \mathrm{Cu}-\mathrm{F}$ can be calculated $a b$ initio with good accuracy. ${ }^{6}$ For example, in the closely related species $\mathrm{C}_{2} \mathrm{H}_{2} \cdots \mathrm{Cu}-\mathrm{Cl}$ calculations at the MP2/cc-pVTZ level of theory reproduced $\Delta_{J}$ and $\Delta_{J K}$ to within a few standard deviations of the well-determined experimental values. Here, the five quartic constants $\left(\Delta_{J}, \Delta_{J K}, \Delta_{K}, \delta_{J}\right.$ and $\left.\delta_{J K}\right)$ for $\mathrm{C}_{2} \mathrm{H}_{2} \cdots \mathrm{Cu}-\mathrm{F}$ were calculated for the optimized geometry at the higher level of theory MP2/aug-cc-pVQZ with the aid of the Gaussian electronic structure package ${ }^{25}$ for each isotopologue investigated. The calculated constants $\Delta_{J}$ and $\Delta_{J K}$ were then fixed in the PGO$\mathrm{PHER}^{23}$ fits of observed frequencies to determine the rotational constants $A_{0}, B_{0}$ and $C_{0}$ and the $\mathrm{Cu}$ nuclear quadrupole coupling constants $\chi_{a a}(\mathrm{Cu})$ and $\chi_{b b}(\mathrm{Cu})-\chi_{c c}(\mathrm{Cu})$ for the isotopologues ${ }^{12} \mathrm{C}_{2} \mathrm{H}_{2} \cdots{ }^{63} \mathrm{Cu}^{19} \mathrm{~F}$ and ${ }^{12} \mathrm{C}_{2} \mathrm{H}_{2} \cdots{ }^{65} \mathrm{Cu}^{19} \mathrm{~F}$ in the first instance.

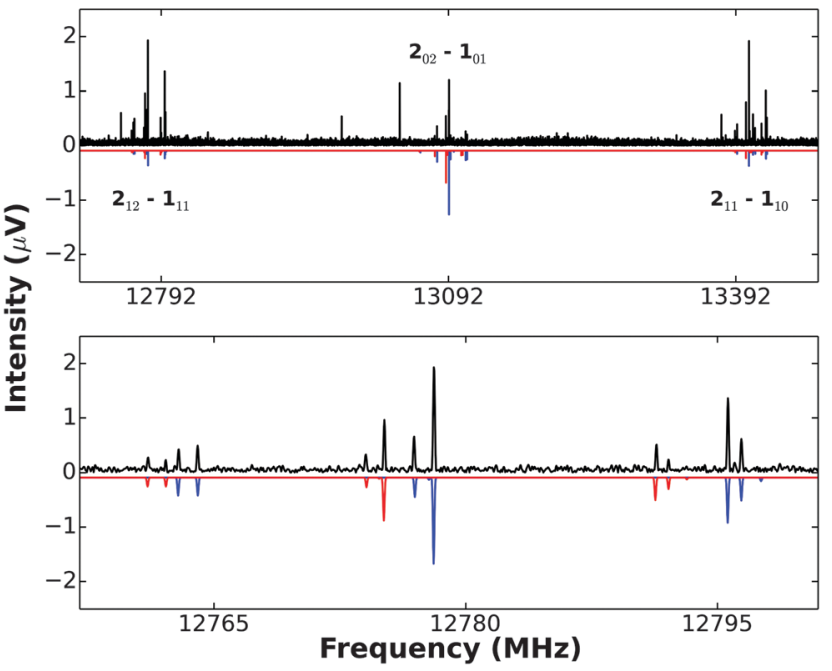

Fig. 2 A recording (10 $\times$ vertical magnification) of the three $J=2 \rightarrow 1$ transitions of ${ }^{12} \mathrm{C}_{2} \mathrm{H}_{2} \cdot{ }^{63} \mathrm{Cu}^{19} \mathrm{~F}$ and ${ }^{12} \mathrm{C}_{2} \mathrm{H}_{2} \cdot{ }^{65} \mathrm{Cu}^{19} \mathrm{~F}$, showing $\mathrm{Cu}$ nuclear quadrupole hyperfine structure (upper panel). The downward pointing spectrum is that synthesized by using PGOPHER and the spectroscopic constants given in Table 1 ( $2 \mathrm{~K}$ rotational temperature assumed). This simulation does not include nuclear spin statistics, so the difference between the simulation and observed spectrum shows clearly the presence of nuclear spin statistics in the latter. Even though hyperfine splitting is greater in the two outer transitions $2_{12} \rightarrow 1_{11}$ and $2_{11} \rightarrow 1_{10}$, their statistical weight advantage $(3: 1)$ over the central $2_{02} \rightarrow 1_{01}$ transition is evident. The lower panel gives an expanded version of the $2_{12} \rightarrow 1_{11}$ transitions of ${ }^{12} \mathrm{C}_{2} \mathrm{H}_{2} \cdot{ }^{63} \mathrm{Cu}^{19} \mathrm{~F}$ (blue) and ${ }^{12} \mathrm{C}_{2} \mathrm{H}_{2} \cdots{ }^{65} \mathrm{Cu}^{19} \mathrm{~F}$ (red). The simulated spectrum (with appropriate scaling of intensities) is again downward pointing.

The spectroscopic constants so determined both from measurements made with the Balle-Flygare and the chirped-pulse spectrometers are displayed in Table 1 . The residuals of the fit to frequencies collected with the Balle-Flygare spectrometer were poorer than the usual few $\mathrm{kHz}$. This arises because $\mathrm{C}_{2} \mathrm{H}_{2} \cdots \mathrm{CuF}$ carries four nuclei $(\mathrm{H}, \mathrm{H}, \mathrm{Cu}$ and $\mathrm{F}$ ) with magnetic moments of significant magnitude, in addition to the electric quadrupolar moment of the $\mathrm{Cu}$ nucleus. Moreover, only the lowest $J$ transitions were available because of the relatively large rotational constants. The magnetic coupling of these nuclei (Cu, F spin-spin coupling, for example) can lead to further complicated but minor, partially resolved splitting or broadening and/or lineshape distortion of each $\mathrm{Cu}$ nuclear quadrupole component and is most significant at the lowest $J$. Therefore assignment of the magnetic sub-structure was not possible in the observed transitions of $\mathrm{C}_{2} \mathrm{H}_{2} \cdots \mathrm{CuF}$, particularly those with $K_{-1}=1$, and hence larger than normal residuals in the fit of the $\mathrm{Cu}$ nuclear quadrupole hyperfine structure resulted. When measured with the lower resolution, chirped-pulse instrument, fitted frequencies assigned to $\mathrm{Cu}$ quadrupole components gave similar magnitude residuals. Reassuringly, the spectroscopic constants determined independently with the two spectrometers are identical within experimental error.

Measurements for ${ }^{13} \mathrm{C}_{2} \mathrm{H}_{2} \ldots{ }^{63,65} \mathrm{Cu}^{19} \mathrm{~F}$, and ${ }^{12} \mathrm{C}_{2} \mathrm{D}_{2} \ldots{ }^{63,65} \mathrm{Cu}^{19} \mathrm{~F}$ were made exclusively on the chirped-pulse instrument to conserve the more expensive isotopic materials. The spectroscopic constants 
Table 1 Ground-state spectroscopic constants of ${ }^{12} \mathrm{C}_{2} \mathrm{H}_{2} \cdot{ }^{63,65} \mathrm{CuF}$ as determined with two different pulsed-jet, Fourier-transform microwave spectrometers (Balle-Flygare and chirped-pulse types)

\begin{tabular}{|c|c|c|c|c|}
\hline \multirow[b]{2}{*}{ Spectroscopic constant } & \multicolumn{2}{|l|}{ Balle-Flygare } & \multicolumn{2}{|l|}{ Chirped-pulse } \\
\hline & ${ }^{12} \mathrm{C}_{2} \mathrm{H}_{2} \cdots{ }^{63} \mathrm{CuF}$ & ${ }^{12} \mathrm{C}_{2} \mathrm{H}_{2} \cdots{ }^{65} \mathrm{CuF}$ & ${ }^{12} \mathrm{C}_{2} \mathrm{H}_{2} \ldots{ }^{63} \mathrm{CuF}$ & ${ }^{12} \mathrm{C}_{2} \mathrm{H}_{2} \cdots{ }^{65} \mathrm{CuF}$ \\
\hline$A_{0} / \mathrm{MHz}$ & $34749(83)$ & $34930(270)$ & $35030(230)$ & $35020(180)$ \\
\hline$B_{0} / \mathrm{MHz}$ & $3431.4407(48)$ & $3430.5201(55)$ & $3431.4404(56)$ & $3430.5159(42)$ \\
\hline$C_{0} / \mathrm{MHz}$ & $3116.7391(44)$ & $3115.9722(55)$ & $3116.7348(56)$ & $3115.9752(39)$ \\
\hline$\Delta_{J K} / \mathrm{kHz}^{a}$ & [38.3] & [38.2] & [38.3] & [38.2] \\
\hline$\Delta_{\mathrm{J}} / \mathrm{kHz}^{a}$ & {$[0.61]$} & {$[0.61]$} & {$[0.61]$} & {$[0.61]$} \\
\hline$\chi_{a a}(\mathrm{Cu}) / \mathrm{MHz}$ & $69.991(47)$ & $64.721(53)$ & $69.979(53)$ & $64.720(40)$ \\
\hline$\left\{\chi_{b b}(\mathrm{Cu})-\chi_{c c}(\mathrm{Cu})\right\} / \mathrm{MHz}$ & $-79.17(11)$ & $-73.25(12)$ & $-79.17(13)$ & $-73.224(73)$ \\
\hline & 20 & 16 & 18 & 19 \\
\hline$\sigma_{\text {r.m.s. }} / \mathrm{kHz}^{c}$ & 30 & 33 & 34 & 26 \\
\hline
\end{tabular}

${ }^{a}$ Values calculated at the MP2/aug-cc-pVQZ level of theory and fixed in the fit. ${ }^{b}$ Number of nuclear quadrupole components included in the fit. ${ }^{c}$ Standard deviation of fit.

for these species were obtained in a similar way and are recorded in Table 2. Detailed fits are available as Supplementary Data ${ }^{26}$ at http://dx.doi.org/xxxxx. The effect of changes in the assumed values of the centrifugal distortion constants were investigated for the isotopologue ${ }^{12} \mathrm{C}_{2} \mathrm{H}_{2} \cdots{ }^{63} \mathrm{Cu}^{19} \mathrm{~F}$. Changes of $10 \%$ in the assumed values of the distortion constants $\Delta_{I}$ and $\Delta_{I K}$ lead to changes in $A$ that are small compared with the errors shown in Table 1 . The standard deviations in $A_{0}$ values are higher than those identified in other B $\cdots$ MX studies because only one $J+1 \rightarrow J$ transition is available within the bandwidth of the spectrometer in the present work.

It is significant that values of the $A_{0}$ for ${ }^{12} \mathrm{C}_{2} \mathrm{H}_{2} \cdots{ }^{63} \mathrm{Cu}^{19} \mathrm{~F}$ and ${ }^{12} \mathrm{C}_{2} \mathrm{H}_{2} \cdots{ }^{65} \mathrm{Cu}^{19} \mathrm{~F}$ (Table 1) are equal within experimental error (as required if $\mathrm{Cu}$ lies on the $a$ axis). These values of $A_{0}$ and their associated uncertainties strongly imply that the average of the two results provides the most reliable value for this quantity, namely $34840(90) \mathrm{MHz}$ (where the quoted error is the range about the mean). This value will be used in geometry determinations set out in Section 3.2, together with the mean value $A_{0}=33100(150) \mathrm{MHz}$ for the pair $\left({ }^{13} \mathrm{C}_{2} \mathrm{H}_{2} \cdots{ }^{63} \mathrm{Cu}^{19} \mathrm{~F}\right.$, $\left.{ }^{13} \mathrm{C}_{2} \mathrm{H}_{2} \ldots{ }^{65} \mathrm{Cu}^{19} \mathrm{~F}\right)$ and $A_{0}=25182(26) \mathrm{MHz}$ for the pair $\left({ }^{12} \mathrm{C}_{2} \mathrm{D}_{2} \cdots{ }^{63} \mathrm{Cu}^{19} \mathrm{~F},{ }^{12} \mathrm{C}_{2} \mathrm{D}_{2} \cdots{ }^{65} \mathrm{Cu}^{19} \mathrm{~F}\right)$. We note that $A_{0}=$ $34840(90) \mathrm{MHz}$ is smaller by $435(90) \mathrm{MHz}$ than the $B_{0}$ value of free ${ }^{12} \mathrm{C}_{2} \mathrm{H}_{2}$ (see Table 3 for various properties of isotopologues of the free molecules ethyne ${ }^{27,28}$ and cuprous fluoride ${ }^{22}$ ). Similar conclusions (see Table 2) apply to the pair $\left({ }^{13} \mathrm{C}_{2} \mathrm{H}_{2} \cdots{ }^{63} \mathrm{Cu}^{19} \mathrm{~F}\right.$,
${ }^{13} \mathrm{C}_{2} \mathrm{H}_{2} \cdots{ }^{65} \mathrm{Cu}^{19} \mathrm{~F}$ ), for which the mean decrease from free ${ }^{13} \mathrm{C}_{2} \mathrm{H}_{2}$ is $464(150) \mathrm{MHz}$, and the pair $\left({ }^{12} \mathrm{C}_{2} \mathrm{D}_{2} \cdots{ }^{63} \mathrm{Cu}^{19} \mathrm{~F}\right.$, ${ }^{12} \mathrm{C}_{2} \mathrm{D}_{2} \cdots{ }^{65} \mathrm{Cu}^{19} \mathrm{~F}$ ), for which a mean decrease of $236(26) \mathrm{MHz}$ relative to $B_{0}$ of free ${ }^{12} \mathrm{C}_{2} \mathrm{D}_{2}$ is observed. These decreases, although not well determined, indicate a change in the ethyne geometry when it becomes attached to CuF. Moreover, the very small change in all three rotational constants on substitution of ${ }^{63} \mathrm{Cu}$ by ${ }^{65} \mathrm{Cu}$ establishes that the $\mathrm{Cu}$ atom lies close to the centre of mass and therefore that the order along the $a$ axis is ${ }^{*}-\mathrm{Cu}-\mathrm{F}$, where * indicates the centre of the $\mathrm{C} \equiv \mathrm{C}$ bond.

The $\mathrm{Cu}$ nuclear quadrupole coupling constants are reasonably well determined. The ratio of the values $\chi_{a a}\left({ }^{63} \mathrm{Cu}\right) / \chi_{a a}\left({ }^{65} \mathrm{Cu}\right)=$ 1.0814(16) lies within experimental error of the known ratio ${ }^{29}$ $Q\left({ }^{63} \mathrm{Cu}\right) / Q\left({ }^{65} \mathrm{Cu}\right)=1.0806(3)$ of the nuclear electric quadrupole moments of the copper nuclides. The large difference between $\chi_{b b}(\mathrm{Cu})=-74.58 \mathrm{MHz}$ and $\chi_{c c}(\mathrm{Cu})=4.59 \mathrm{MHz}$ is evidence of a large anisotropy in the electric field gradient at $\mathrm{Cu}$ along the $b$ and $c$ principal inertia axis directions arising from the facts that (1) the $\mathrm{Cu}$ atom is close to the ethyne $\pi$ bond and (2) the electron distribution within the triple bond $\mathrm{C} \equiv \mathrm{C}$ is different along the two directions. Indeed, the large difference $\left\{\chi_{b b}\left({ }^{63} \mathrm{Cu}\right)-\chi_{c c}\left({ }^{63} \mathrm{Cu}\right)\right\}=$ $-79.17(11) \mathrm{MHz}$ provides convincing evidence of the proximity of $\mathrm{Cu}$ to the $\mathrm{C} \equiv \mathrm{C}$ bond. In the related molecule $\mathrm{C}_{2} \mathrm{H}_{2} \cdots \mathrm{Ag}-\mathrm{Cl}$, in which $\mathrm{Cl}$ is remote from the ethyne $\pi$ bond, values of the $\mathrm{Cl}$ nuclear quadrupole coupling constants are $\chi_{a a}\left({ }^{35} \mathrm{Cl}\right)=-28.9268(78) \mathrm{MHz}$ and $\left\{\chi_{b b}\left({ }^{35} \mathrm{Cl}\right)-\chi_{c c}\left({ }^{35} \mathrm{Cl}\right)\right\}=-3.256(7) \mathrm{MHz}$.

Table 2 Ground-state spectroscopic constants of ${ }^{13} \mathrm{C}_{2} \mathrm{H}_{2} \ldots{ }^{63,65} \mathrm{CuF}$ and ${ }^{12} \mathrm{C}_{2} \mathrm{D}_{2} \ldots{ }^{63.65} \mathrm{CuF}$ as determined with a chirped-pulse, pulsed-jet, Fouriertransform microwave spectrometer

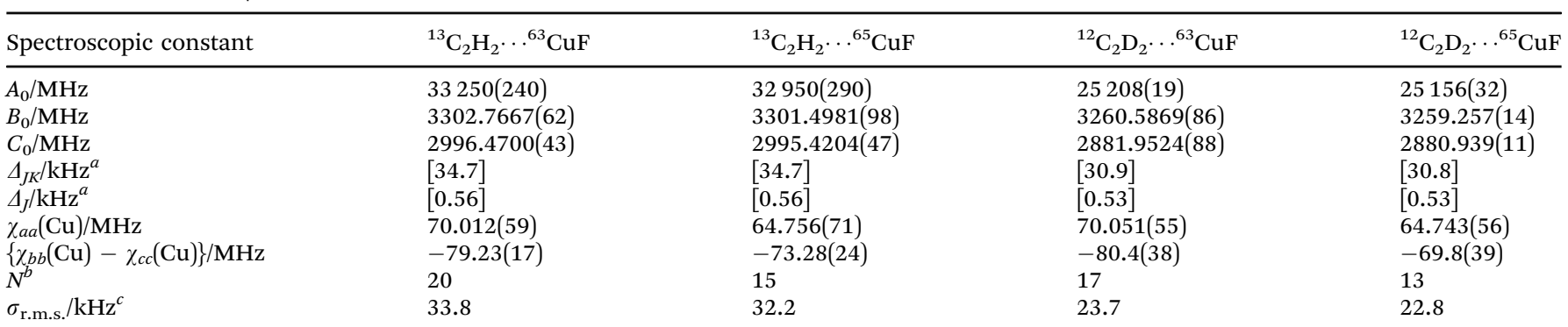

${ }^{a}$ Values calculated at the MP2/aug-cc-pVQZ level of theory and fixed in the fit. ${ }^{b}$ Number of nuclear quadrupole components included in the fit.

${ }^{c}$ Standard deviation of fit. 
Table 3 Some spectroscopic constants and bond lengths of $\mathrm{C}_{2} \mathrm{H}_{2}$ and CuF referred to in evaluating properties of $\mathrm{C}_{2} \mathrm{H}_{2} \cdots \mathrm{CuF}$

\begin{tabular}{|c|c|c|c|c|c|}
\hline Quantity constant & ${ }^{12} \mathrm{C}_{2} \mathrm{H}_{2}$ & ${ }^{13} \mathrm{C}_{2} \mathrm{H}_{2}$ & ${ }^{12} \mathrm{C}_{2} \mathrm{D}_{2}$ & ${ }^{63} \mathrm{Cu}^{19} \mathrm{~F}$ & ${ }^{65} \mathrm{Cu}^{19} \mathrm{~F}$ \\
\hline Cuprous fluoride & & & & - & - \\
\hline$r_{0}(\mathrm{Cu}-\mathrm{F}) / \AA$ & - & - & - & $1.74865^{f}$ & $1.74863^{f}$ \\
\hline $\begin{array}{l}r_{\mathrm{e}}(\mathrm{Cu}-\mathrm{F}) / \AA \text { (experiment) } \\
\left.r_{\mathrm{e}}(\mathrm{Cu}-\mathrm{F}) / \AA \text { ( } a b \text { initio calc. }\right)\end{array}$ & - & $\overline{-}$ & - & $\begin{array}{l}1.74493^{g} \\
1.7430(3)^{h}\end{array}$ & $1.74493^{g}$ \\
\hline
\end{tabular}

${ }^{a}$ Ref. 27. ${ }^{b}$ Calculated by fitting the moments of inertia $I_{b}^{0}$ of ${ }^{12} \mathrm{C}_{2} \mathrm{H}_{2}$ and ${ }^{13} \mathrm{C}_{2} \mathrm{H}_{2}$ from ref. 27. See text. ${ }^{c}$ Calculated by fitting the moment of inertia $I_{b}^{0}$ of ${ }^{12} \mathrm{C}_{2} \mathrm{D}_{2}$ with $r(\mathrm{C} \equiv \mathrm{C})$ fixed at $1.206553 \AA{ }^{d}{ }^{d}$ Ref. 28. ${ }^{e}$ Ref. $22 .{ }^{f}$ Calculated from $B_{0}$ value by using $r_{0}=\left\{h / 8 \pi^{2} \mu B_{0}\right\}^{\frac{1}{2}}$, with $B_{0}$ estimated from Dunham constants in ref. $22 .{ }^{g}$ Calculated from $B_{\mathrm{e}}$ value by using $r_{\mathrm{e}}=\left\{h / 8 \pi^{2} \mu B_{\mathrm{e}}\right\}^{\frac{1}{2}}$ with $B_{\mathrm{e}}$ from ref. $22 .{ }^{h} \mathrm{Calculated}$ at the CCSD(T)(F12*)/aug-ccpwCVQZ level of theory, with post-CCSD(T) corrections made for basis set incompleteness, core-valence correlation, and higher-order correlations.

\subsection{Molecular geometry}

Of the four consequences of a planar geometry of the type shown in Fig. 1, it was indicated in Section 3.1 that three were satisfied by experimental results for $\mathrm{C}_{2} \mathrm{H}_{2} \cdots \mathrm{Cu}-\mathrm{F}$. The fourth is that the inertia defect, as defined in eqn (2), should be small and positive. If the $\mathrm{Cu}$ atom does lie on the $a$ axis, it is reasonable to use the mean $I_{a}^{0}$ value for a pair of isotopologues ${ }^{m} \mathrm{C}_{2}{ }^{n} \mathrm{H}_{2} \cdots{ }^{63} \mathrm{Cu}^{19} \mathrm{~F}$ and ${ }^{m} \mathrm{C}_{2}{ }^{n} \mathrm{H}_{2} \cdots{ }^{65} \mathrm{Cu}^{19} \mathrm{~F}$ in calculating $\Delta_{0}$ for both members of the pair. The results are $\Delta_{0}=0.365(38) \mathrm{u} \AA^{2}$ and 0.366(38) u $\AA^{2}$ for the ${ }^{12} \mathrm{C}_{2} \mathrm{H}_{2} \cdot{ }^{63,65} \mathrm{Cu}^{19} \mathrm{~F}$ pair, 0.373(69) $\mathrm{u} \AA^{2}$ and $0.373(69) \mathrm{u} \AA^{2}$ for the ${ }^{13} \mathrm{C}_{2} \mathrm{H}_{2} \cdots{ }^{63,65} \mathrm{Cu}^{19} \mathrm{~F}$ pair, and $0.295(22)$ $\mathrm{u} \AA^{2}$ and $0.293(22) \mathrm{u} \AA^{2}$ for the ${ }^{12} \mathrm{C}_{2} \mathrm{D}_{2} \cdot{ }^{63,65} \mathrm{Cu}^{19} \mathrm{~F}$ pair. The very similar values, observed across a similar range of isotopologues, for each of the related planar molecules $\mathrm{C}_{2} \mathrm{H}_{2} \cdots \mathrm{Cu}^{-\mathrm{Cl}^{6}}$ and $\mathrm{C}_{2} \mathrm{H}_{2} \cdots \mathrm{Ag}-\mathrm{Cl}^{5}$ reinforce the conclusion that $\mathrm{C}_{2} \mathrm{H}_{2} \cdots \mathrm{Cu}-\mathrm{F}$ is also planar.

Thus, all the evidence so far presented is consistent with a geometry of $\mathrm{C}_{2} \mathrm{H}_{2} \cdots \mathrm{CuF}$ of the planar, T-shaped type of $C_{2 \mathrm{v}}$ symmetry illustrated in Fig. 1, with the atoms in the indicated order. Quantitative detail of the geometry can be determined experimentally in two ways from the zero-point rotational constants of the six isotopologues of $\mathrm{C}_{2} \mathrm{H}_{2} \cdots \mathrm{CuF}$ investigated, namely a partial $r_{\mathrm{s}}$ geometry and an almost complete $r_{0}$ geometry. These results will be compared with the full $r_{\mathrm{e}}$ geometry obtained from $a b$ initio calculations carried out at the $\operatorname{CCSD}(\mathrm{T})(\mathrm{F} 12 * / \mathrm{AVTZ})$ level of theory.

If equilibrium rotational constants (and therefore equilibrium principal moments of inertia) of the various isotopologues of $\mathrm{C}_{2} \mathrm{H}_{2} \cdots \mathrm{CuF}$ investigated were available, the equilibrium principalaxis co-ordinates $a_{\mathrm{C}}, b_{\mathrm{C}}, a_{\mathrm{H}}$, and $a_{\mathrm{Cu}}$ could be determined by means of eqn (3)-(5). In view of the molecular planarity and the existence of the symmetry operation $C_{2}^{\mathrm{a}}$, the changes $I_{b}$ and $I_{a}$ in the equilibrium principal moments of inertia that accompany the double isotopic substitution of $\mathrm{X}$ by $\mathrm{X}^{\prime}\left(\mathrm{X}={ }^{12} \mathrm{C}\right.$ or $\mathrm{H} ; \mathrm{X}^{\prime}={ }^{13} \mathrm{C}$ or $\mathrm{D}$, respectively) at symmetrically equivalent atoms are related to the equilibrium coordinates $a_{\mathrm{X}}$ and $b_{\mathrm{X}}$ by the expressions put forward by Chutjian ${ }^{30}$

$$
\left|a_{\mathrm{X}}\right|=\left(\Delta I_{b} / \mu_{\mathrm{D}}\right)^{\frac{1}{2}}
$$

$$
\left|b_{\mathrm{X}}\right|=\left\{\Delta I_{a} /(2 \Delta m)\right\}^{\frac{1}{2}}
$$

in which $\mu_{\mathrm{D}}=(2 \Delta m M) /(M+2 \Delta m)$ is the reduced mass for the double substitution in the parent isotopologue of mass $M$ leading to a mass change $2 \Delta m$. The equilibrium principal axis co-ordinate $a_{\mathrm{Cu}}$ of the atom $\mathrm{Cu}$, which lies on the $a$-axis of $\mathrm{C}_{2} \mathrm{H}_{2} \cdots \mathrm{CuF}$, is given in terms of changes $\Delta I_{b}$ and $\Delta I_{c}$ in equilibrium principal moments of inertia accompanying isotopic substitution at $\mathrm{Cu}$ by Kraitchman's equation ${ }^{31}$

$$
\left|a_{\mathrm{Cu}}\right|=\left\{\left(\Delta I_{b}+\Delta I_{c}\right) / 2 \mu_{\mathrm{s}}\right\}^{\frac{1}{2}}
$$

where $\mu_{\mathrm{S}}=(\Delta m M) /(M+\Delta m)$ is the reduced mass for the substitution. When equilibrium quantities are unavailable, Costain $^{32,33}$ proposed using zero-point $\Delta I^{0}$ values in place of their equilibrium counterparts $\Delta I^{\mathrm{e}}$ in eqn (3)-(5) and named the resulting coordinates as substitution or $r_{\mathrm{s}}$ coordinates. The $r_{\mathrm{s}}$ coordinates $a_{\mathrm{C}}, b_{\mathrm{C}}, a_{\mathrm{H}}, b_{\mathrm{H}}$ and $a_{\mathrm{Cu}}$ so obtained from eqn (3)-(5) are given in Table 4 . Each refers to the principal inertia axes of ${ }^{12} \mathrm{C}_{2} \mathrm{H}_{2} \cdots{ }^{63} \mathrm{Cu}^{19} \mathrm{~F}$ as the parent molecule and the chosen signs are those that lead to reasonable bond lengths. Note that the mean of $A_{0}$ for pairs of ${ }^{63,65} \mathrm{Cu}$ isotopologues, as given in Section 3.1, were used in the calculation of the $b_{\mathrm{C}}$ and $b_{\mathrm{H}}$ coordinates. The substantial uncertainties in $b_{\mathrm{C}}$ and $b_{\mathrm{H}}$ result from the relatively large errors assigned to $A_{0}$. A $r_{\mathrm{s}}$ coordinate $a_{\mathrm{F}}$ is not available because fluorine possesses only one stable isotope and so the value of $a_{\mathrm{F}}$ given in Table 4 was determined, as recommended by Costain, ${ }^{32}$ from the other $r_{\mathrm{s}}$ coordinates with the aid of the first moment condition,

$$
a_{\mathrm{F}}=-\left(\sum_{j} m_{j} a_{j}\right) / m_{\mathrm{F}},
$$

in which the sum over $j$ indicates that all atoms but $\mathrm{F}$ are included.

The significantly larger magnitude of $a_{\mathrm{H}}$ than $a_{\mathrm{C}}$ provides experimental evidence that the angular geometry of ethyne is distorted in the manner shown in Fig. 1, that is the $\mathrm{H}$ atoms move away from the $\mathrm{C} \equiv \mathrm{C}$ internuclear line when the complex is formed, with the two equivalent angles $A=\angle \mathrm{H}-\mathrm{C} \equiv{ }^{*}$ (see Fig. 1 for definition) exceeding $180^{\circ}$. The value obtained from 
Table 4 Various types of principal inertia axis coordinate and geometry determined for $\mathrm{C}_{2} \mathrm{H}_{2} \cdots \mathrm{Cu}-\mathrm{F}$

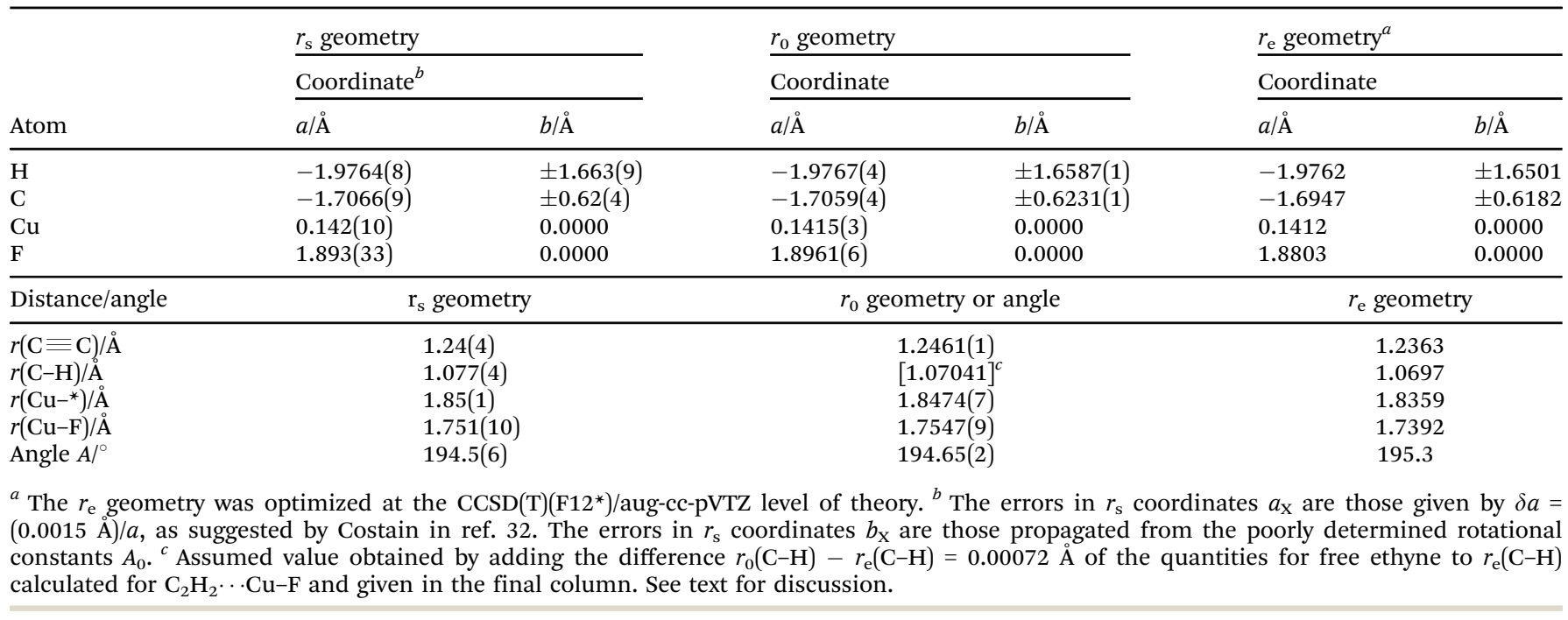

the $r_{\mathrm{s}}$ coordinates in Table 3 is $A=194.5(6)^{\circ}$ (see Table 4 ), where the large error is consequent upon the errors in the $A_{0}$ values. This distortion is established with greater accuracy in the experimental $r_{0}$ and $a b$ initio $r_{\mathrm{e}}$ geometries discussed later (see below). The bond distances implied by the $r_{\mathrm{s}}$ coordinates are also included in Table 4.

When the minimum number of bond lengths and angles required to establish a molecular geometry uniquely is fitted to a sufficient number of zero-point principal moments of inertia by the least-squares method, the result is called a $r_{0}$ geometry, i.e one obtained when the rovibrational contributions $\varepsilon_{a}=I_{a}^{0}-I$ $\underset{a}{\mathrm{e}}$, etc. to the moments of inertia are ignored. A fit of $I_{b}^{0}$ and $I_{c}^{0}$ (values of $I_{a}^{0}$ were excluded because of their relatively low accuracy) of all six isotopologues of $\mathrm{C}_{2} \mathrm{H}_{2} \cdots \mathrm{Cu}-\mathrm{F}$ implied by the rotational constants $B_{0}$ and $C_{0}$ reported in Tables 1 and 2 to determine $r\left(\mathrm{C} \equiv{ }^{*}\right), r\left({ }^{*}-\mathrm{Cu}\right), r(\mathrm{Cu}-\mathrm{F}), r(\mathrm{C}-\mathrm{H})$, and $A=\angle \mathrm{H}-\mathrm{C} \equiv{ }^{*}$ was unsatisfactory, however, because of high correlation between two last-named quantities. To break the correlation, the following approach was used. A good estimate of $r_{0}(\mathrm{C}-\mathrm{H})$ is given by adding $r_{\mathrm{e}}(\mathrm{C}-\mathrm{H})$ of $\mathrm{C}_{2} \mathrm{H}_{2} \cdots \mathrm{Cu}-\mathrm{F}$ from the $\mathrm{CCSD}(\mathrm{T})\left(\mathrm{F} 12^{*}\right) / \mathrm{AVTZ}$ calculation to the difference $r_{0}(\mathrm{C}-\mathrm{H})-r_{\mathrm{e}}(\mathrm{C}-\mathrm{H})=0.00072 \AA$ for free ethyne $\mathrm{e}^{27,28}$ (Table 3), with the result $r_{0}(\mathrm{C}-\mathrm{H})=1.07041 \AA$. This value was then fixed in the fit. The geometry and principal axis coordinates were then determined under this assumption with the aid of Kisiel's program STRFIT ${ }^{34}$ and are included in Table 4. There is excellent agreement with the corresponding $r_{\mathrm{s}}$ quantities, given the large error in the $b_{\mathrm{s}}$ coordinates propagated through the rotational constants $A_{0}$.

\section{Discussion}

The new molecule $\mathrm{C}_{2} \mathrm{H}_{2} \cdots \mathrm{Cu}-\mathrm{F}$ has been synthesized by interaction of the plasma produced by laser ablation of a copper rod with a supersonically-expanded pulse of gas containing $\mathrm{SF}_{6}$ and $\mathrm{C}_{2} \mathrm{H}_{2}$ diluted with a large excess of argon. It was detected by means of its ground-state rotational spectrum and was shown to have a planar,
T-shaped geometry of $C_{2 \mathrm{v}}$ symmetry, with the CuF molecule lying along a $C_{2}$ axis of the $\mathrm{C}_{2} \mathrm{H}_{2}$ molecule. Quantitative aspects of the molecular geometry were determined through the investigation of six isotopologues. A significant finding is that the ethyne subunit is considerably distorted by the interaction of its $\mathrm{C} \equiv \mathrm{C}$ bond with the $\mathrm{Cu}$ atom of $\mathrm{CuF}$. The $\mathrm{H}$ atoms move from the $\mathrm{C} \equiv \mathrm{C}$ internuclear line, in the direction away from the approaching $\mathrm{Cu}$ atom, so that the ${ }^{*} \equiv \mathrm{C}-\mathrm{H}$ angle increases from $180^{\circ}$ to $194.65(2)^{\circ}$. The $\mathrm{C} \equiv \mathrm{C}$ bond lengthens by $c a$. $0.04 \AA$. These distortions are well reproduced in the optimized geometry of $\mathrm{C}_{2} \mathrm{H}_{2} \cdots \mathrm{Cu}-\mathrm{F}$ obtained from $a b$ initio calculations carried out at the $\operatorname{CCSD}(\mathrm{T})\left(\mathrm{F} 12^{*}\right) / \mathrm{AVTZ}$ level of theory (see Table 4).

Similar distortions have now been observed in the series of molecules $\mathrm{C}_{2} \mathrm{H}_{2} \cdots \mathrm{CuF}, \mathrm{C}_{2} \mathrm{H}_{2} \cdots \mathrm{AgCl},{ }^{4} \mathrm{C}_{2} \mathrm{H}_{2} \cdots \mathrm{AgCCH},{ }^{5}$ and $\mathrm{C}_{2} \mathrm{H}_{2} \cdots \mathrm{CuCl}^{6}{ }^{6}$ each of which has the planar T-shaped, $C_{2 \mathrm{v}}$ geometry with the metal atom adjacent to the ethyne $\pi$ bond (see Fig. 1). The changes $\delta A$ in the angle ${ }^{*} \equiv \mathrm{C}-\mathrm{H}$ and $\delta r$ in the distance $r(\mathrm{C} \equiv \mathrm{C})$ are $\left[14.65(2)^{\circ}, 4.0(1) \mathrm{pm}\right],\left[7.70(4)^{\circ}, 2.86(4)\right.$ $\mathrm{pm}],\left[5.9(1)^{\circ}, 2.61(3) \mathrm{pm}\right]$ and $\left[12.5(2)^{\circ}, 2.7(3) \mathrm{pm}\right]$ respectively, along the series. The corresponding energy changes $D_{\mathrm{e}}$ accompanying dissociation into $\mathrm{C}_{2} \mathrm{H}_{2}$ and $\mathrm{MX}$ have been calculated ab initio [at the $\operatorname{CCSD}(\mathrm{T})\left(\mathrm{F} 12^{*}\right) / \mathrm{AVTZ}$ or AVDZ levels] to be 170 , 97.5, 92 and $148 \mathrm{~kJ} \mathrm{~mol}^{-1}$, respectively. Fig. 3 displays a plot of each of $\delta A$ and $\delta r$ versus $D_{\mathrm{e}}$ for these molecules. The origin has been included as a point in each plot because when the interaction energy $D_{\mathrm{e}}$ is zero, presumably there is no distortion of the ethyne subunit. Fig. 3 shows that there is an approximate linear relationship between each of the two types of distortion $\delta A$ and $\delta r$ and the strength of the interaction $D_{\mathrm{e}}$.

The nature of the ethyne distortions can be understood with the aid of a simple model. The nuclei of each of the $\mathrm{C}_{2} \mathrm{H}_{2} \cdots \mathrm{M}-\mathrm{X}$ $(\mathrm{M}=\mathrm{Cu}$ or $\mathrm{Ag}, \mathrm{X}=\mathrm{F}$ or $\mathrm{Cl})$ molecules all lie in the $a b$ principal inertia plane. Let the symmetry plane of one of the ethyne $\pi$ bonding orbitals lie in this plane, with that of the other $\pi$ orbital perpendicular to it. When $\mathrm{M}-\mathrm{X}$ approaches from large separation and takes up its position along a $C_{2}$ axis of ethyne (which then becomes the $a$ axis of the new molecule), the partial 


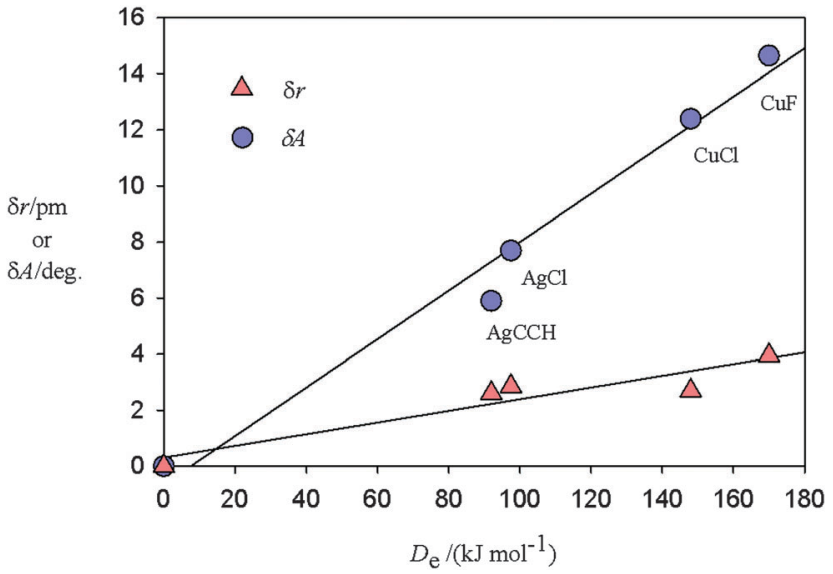

Fig. 3 Plots of the angular distortion $\delta A$ (see Fig. 1 for a definition of the angle $\delta A$ ) and the extension $\delta r$ of the $\mathrm{C} \equiv \mathrm{C}$ bond of ethyne on formation of the complexes $\mathrm{C}_{2} \mathrm{H}_{2} \cdots M X$ against the energy $D_{\mathrm{e}}$ required for the dissociation reaction $\mathrm{C}_{2} \mathrm{H}_{2} \cdots M X=\mathrm{C}_{2} \mathrm{H}_{2}+M X$. $\delta A$ and $\delta r$ are experimentally determined while the $D_{\mathrm{e}}$ values are calculated $a b$ initio at the $\operatorname{CCSD}(\mathrm{T})\left(\mathrm{F} 12^{*}\right) / A V T Z$ or AVDZ level of theory. The origin is included as a point under the assumption that zero interaction energy is associated with $\delta A=0$ and $\delta r=0$.

positive charge $\delta+$ that exists on the M atom in the species $\mathrm{M}-\mathrm{X}$ (the metal halides have large electric dipole moments in the range $\sim 5$ or $6 \mathrm{D})^{35}$ polarizes electron density preferentially from the $\pi$ orbital $\left(\pi_{a b}\right)$ in the molecular plane. The larger the polarization, the more the $\pi$ bonding in the ethyne molecule will resemble that of an ethene molecule in which the symmetry plane of its $\pi$ bonding orbital is perpendicular to the $a b$ plane and the $\mathrm{C}-\mathrm{H}$ bonds lie in the $a b$ plane. In the limit of very large polarization of the $\pi_{a b}$ electrons, when both are completely removed from ethyne to yield the ion $\mathrm{C}_{2} \mathrm{H}_{2}{ }^{2+}$, the distortions would be of the order $\delta A \approx 60^{\circ}$ and $\delta r \approx 134.5-120.6=$ $13.9 \mathrm{pm}$, where 134.5 and $120.6 \mathrm{pm}$ are the CC distances in the ground state ${ }^{3} \Sigma_{\mathrm{g}}{ }^{-}$of the dication ${ }^{36} \mathrm{C}_{2} \mathrm{H}_{2}{ }^{2+}$ and in ethyne, ${ }^{27}$ respectively. Similar distortions have been observed in $\mathrm{C}_{2} \mathrm{H}_{4} \cdots \mathrm{M}-\mathrm{X}(\mathrm{M}=$ $\mathrm{Cu}$ or $\mathrm{Ag}$ ), ${ }^{6,37}$ in which $\mathrm{M}-\mathrm{X}$ lies on the $\mathrm{C}_{2}$ axis of $\mathrm{C}_{2} \mathrm{H}_{4}$ that is perpendicular to the plane of the $\mathrm{C}_{2} \mathrm{H}_{4}$ nuclei. Similar arguments can be used to rationalize the distortions in these molecules, but with $\mathrm{C}_{2} \mathrm{H}_{4}{ }^{2+}$ as the limiting dication.

\section{Acknowledgements}

We thank the European Research Council for postdoctoral fellowships awarded to D.P.Z. and S.L.S., and for project funding (CPFTMW-307000). ACL thanks the University of Bristol for a Senior Research Fellowship and Newcastle University for a Faculty of SAgE Visiting Professorship. D.P.Z. thanks Newcastle University for the award of a Faculty of SAgE Research Fellowship. D.P.T. gratefully acknowledges the award by the Royal Society of a University Research Fellowship.

\section{References}

1 K. Schroeter, C. A. Schalley, R. Wesendrup, D. Schroder and H. Schwarz, Organometallics, 1997, 16, 986.
2 K. Judai, A. S. Worz, S. Abbet, J. M. Antonietti, U. Heiz, A. Del Vitto, L. Giordano and G. Pacchioni, Phys. Chem. Chem. Phys, 2005, 7, 955.

3 A. Molnar, A. Sarkany and M. Varga, J. Mol. Catal. A: Chem., 2001, 173, 185.

4 S. L. Stephens, W. Mizukami, D. P. Tew, N. R. Walker and A. C. Legon, J. Chem. Phys., 2012, 137, 174302.

5 S. L. Stephens, D. P. Zaleski, W. Mizukami, D. P. Tew, N. R. Walker and A. C. Legon, J. Chem. Phys., 2014, 140, 124310.

6 S. L. Stephens, D. M. Bittner, V. A. Mikhailov, W. Mizukami, D. P. Tew, N. R. Walker and A. C. Legon, Inorg. Chem., 2014, 53, 10722-10730.

7 G. G. Brown, B. C. Dian, K. O. Douglass, S. M. Geyer, S. T. Shipman and B. H. Pate, Rev. Sci. Instrum., 2008, 79, 053103.

8 S. L. Stephens and N. R. Walker, J. Mol. Spectrosc., 2010, 263, 27-33.

9 D. P. Zaleski, S. L. Stephens and N. R. Walker, Phys. Chem. Chem. Phys., 2014, 16, 25221-25228.

10 T. J. Balle and W. H. Flygare, Rev. Sci. Instrum., 1981, 52, 33-45.

11 R. C. Batten, G. C. Cole and A. C. Legon, J. Chem. Phys., 2013, 119, 7903-7912.

12 C. Hättig, D. P. Tew and A. Köhn, J. Chem. Phys., 2010, 132, 231102.

13 C. Hättig, W. Klopper, A. Köhn and D. P. Tew, Chem. Rev., 2011, 112, 4-74.

14 K. Raghavachari, G. W. Trucks, J. A. Pople and M. HeadGordon, Chem. Phys. Lett., 1989, 157, 479-483.

15 R. A. Kendall, T. H. Dunning and R. J. Harrison, J. Chem. Phys., 1992, 96, 6796-6806.

16 K. A. Peterson and C. Puzzarini, Theor. Chem. Acc., 2005, 114, 283-296.

17 M. Dolg, U. Wedig, H. Stoll and H. J. Preuss, Chem. Phys., 1987, 86, 866-872.

18 H.-J. Werner, P.J. Knowles, R. Lindh, F.R. Manby and M. Schütz, MOLPRO, version 2009.1, a package of ab initio programs; 2009, see http://www.molpro.net.

19 S. F. Boys and F. Bernardi, Mol. Phys., 1970, 19, 553-566.

20 M. Heckert, M. Kállay and J. Gauss, Mol. Phys., 2005, 103, 2109.

21 M. Kállay, MRCC, a generalized CC/CI program, see http:// www.mrcc.hu.

22 T. Okabayashi, E. Yamazaki, T. Honda and M. Tanimoto, J. Mol. Spectrosc., 2001, 209, 66-70.

23 PGOPHER, a program for simulating rotational structure, designed by C.M. Western, University of Bristol, version 6.0.202, 2010, available at http://pgopher.chm.bris.ac.uk.

24 J. K. G. Watson, J. Chem. Phys., 1968, 46, 1935.

25 M. J. Frisch, G. W. Trucks, H. B. Schlegel, G. E. Scuseria, M. A. Robb, J. R. Cheeseman, G. Scalmani, V. Barone, B. Mennucci, G. A. Petersson, H. Nakatsuji, M. Caricato, X. Li, H. P. Hratchian, A. F. Izmaylov, J. Bloino, G. Zheng, J. L. Sonnenberg, M. Hada, M. Ehara, K. Toyota, R. Fukuda, J. Hasegawa, M. Ishida, T. Nakajima, Y. Honda, O. Kitao, H. Nakai, T. Vreven, J. A. Montgomery Jr., J. E. Peralta, F. Ogliaro, M. Bearpark, J. J. Heyd, E. Brothers, K. N. Kudin, 
V. N. Staroverov, T. Keith, R. Kobayashi, J. Normand, K. Raghavachari, A. Rendell, J. C. Burant, S. S. Iyengar, J. Tomasi, M. Cossi, N. Rega, J. M. Millam, M. Klene, J. E. Knox, J. B. Cross, V. Bakken, C. Adamo, J. Jaramillo, R. Gomperts, R. E. Stratmann, O. Yazyev, A. J. Austin, R. Cammi, C. Pomelli, J. W. Ochterski, R. L. Martin, K. Morokuma, V. G. Zakrzewski, G. A. Voth, P. Salvador, J. J. Dannenberg, S. Dapprich, A. D. Daniels, O. Farkas, J. B. Foresman, J. V. Ortiz, J. Cioslowski and D. J. Fox, Gaussian 09, Revision B.01, Gaussian, Inc., Wallingford CT, 2010.

26 See ESI $\dagger$ for assignments of all observed transitions and the fits used to determine the spectroscopic constants of six isotopologues of $\mathrm{C}_{2} \mathrm{H}_{2} \cdots \mathrm{Cu}-\mathrm{F}$.

27 M. Herman, A. Campargue, M. I. El Idrissi and J. Van der Auwera, J. Phys. Chem. Ref. Data, 2003, 32, 921.
28 J. Lievin, J. Demaison, M. Herman, A. Fayt and C. Puzzarini, J. Chem. Phys., 2011, 134, 064119.

29 W. Gordy and R. L. Cook, Microwave Molecular Spectra, Wiley, New York, 1984, Appendix E.

30 A. Chutjian, J. Mol. Spectrosc., 1964, 14, 361.

31 J. Kraitchman, Am. J. Phys., 1953, 21, 17.

32 C. C. Costain, J. Chem. Phys., 1958, 29, 864.

33 C. C. Costain, Trans. Am. Crystallogr. Assoc., 1966, 2, 157.

34 Z. Kisiel, J. Mol. Spectrosc., 2003, 218, 58-67.

35 NIST Diatomic Spectra Database, www.nist.gov/pml/data/msddi/index.cfm, compiled by F.J. Lovas, E. Tiemann, J.S. Coursey, S.A. Kotochigova, J. Chang, K. Olsen and R.A. Dragoset.

36 R. Thissen, J. Delwiche, J. H. Robbe, D. Duflot, J. P. Flament and J. H. D. Eland, J. Chem. Phys., 1993, 99, 6590.

37 S. L. Stephens, D. P. Tew, V. A. Mikhailov, N. R. Walker and A. C. Legon, J. Chem. Phys., 2011, 135, 024315. 